\title{
Effect of the addition of 3-glycidoxypropyltrimethoxysilane to tetraethoxyorthosilicate-based stone protective coating using $n$-octylamine as a catalyst
}

\author{
DAN LI ${ }^{1}$, FEIGAO XU ${ }^{2, *}$, LI SHAO ${ }^{1}$ and MIN WANG ${ }^{2}$ \\ ${ }^{1}$ School of Environmental and Chemical Engineering, Nanchang University, Nanchang 330031, China \\ ${ }^{2}$ College of Science, Nanchang University, Nanchang 330031, China
}

MS received 9 December 2013; revised 1 April 2014

\begin{abstract}
A tetraethoxyorthosilicate (TEOS)-based stone protective coating containing functional 3-glycidoxypropyltrimethoxysilane (GPTMS) has been prepared in order to reduce gel crack formation during the drying phase using $\boldsymbol{n}$-octylamine as a catalyst. The effect of gel time and viscosity on GPTMS concentration were studied. We have demonstrated that the addition of GPTMS accelerated the gel process and improve viscosity of sol. It was found that GPTMS was chemically incorporated into the gel matrix via $\mathrm{Si}-\mathrm{O}$ bonds by Fourier transform infrared spectroscopy (FTIR) analysis. Nitrogen adsorption-desorption isotherms of xerogels were measured, they showed that the pore size of xerogels decreased with the addition of GPTMS. Atomic force microscopy (AFM) showed the surface roughness increased as content of GPTMS was higher. The Scotch Tape test and the hardness values showed improvement of cohesion and consolidation ability of hybrid sol. The protective performance evaluation of the treated stones with hybrid sol indicated its acid rain resistance.
\end{abstract}

Keywords. Stone consolidation; hybrid gels; GPTMS; $n$-octylamine.

\section{Introduction}

Historical carved stone are mostly made from limestone or marble. ${ }^{1,2}$ For historical carved stone, the principal mechanism of chemical weathering is acidic dissolution of the carbonate stone. ${ }^{1,2}$ Many polymer systems (consolidants and coatings) are currently being used to reduce or delay weathering effects on historic buildings and monuments. ${ }^{3}$ In recent years, various commercial consolidants based on tetraethoxyorthosilicate (TEOS) have been widely used in the treatment of construction materials of historical carved stone for consolidation and conservation of such structures. However, protection of carved stone by using coatings has created serious challenges for surface science and technology. It is well known that drawback of these conservation products is their tendency to form brittle gels during the drying phase. ${ }^{4}$ Cracking is generated by the high capillary pressures supported by the gel network during drying. ${ }^{5}$ Son $e t a l^{6}$ and Kim et $a l^{7}$ have developed an strategy by the addition of nanoparticles and/or (3-glycidoxypropyl) trimethoxysilane (GPTMS) having a flexible segment using dibutyltindilaurate as a catalyst. The addition of nanoparticles and GPTMS provided a crack-free gel after drying and the surface hydrophobicity of the treated decayed granite increased with the addition of nanoparticles and GPTMS, while the gel structure has not been reported.

\footnotetext{
*Author for correspondence (xufeigao@ncu.edu.cn)
}

Recently, Mosquera et $a l^{8}$ has designed an innovative synthesis strategy in which the sol-gel transition occurs in the presence of a surfactant ( $n$-octylamine). The surfactant provided an efficient means of preventing cracking of the gel, as the result of two factors: ${ }^{9}$ (1) coarsening of the gel network, which reduces the capillary pressure; and (2) decreasing surface tension, which also reduces capillary pressure. In addition, $n$-octylamine can also be actted as a basic catalyst of the sol-transition on the substrate surface. ${ }^{10,11}$

In this study, an inorganic-organic hybrid sol was synthesized to protect historic carved stone from weathering by starting from a solution of TEOS precursor using GPTMS as a flexible segment in the presence of $n$-octylamine as a catalyst. The GPTMS- $\mathrm{SiO}_{2}$ xerogels structure was tested by using Fourier transform infrared spectroscopy (FTIR), atomic force microscopy (AFM), thermogravimetry analysis (TGA) and $\mathrm{N}_{2}$-adsorption/desorption techniques when $n$-octylamine is used as catalyst.

\section{Experimental}

\subsection{Materials}

TEOS (analytical grade reagent, obtained from Sinopharm Chemical Reagent Corporation). GPTMS, viscosity $\approx 3.4$ $\mathrm{mPa} \mathrm{s} \mathrm{s}^{-1}$ was obtained from Hangzhou Silong Chemical Reagent Corporation, China. The surfactant was $n$-octylamine 
(Aldrich) as a polycondensation catalyst. All reagents were used as received without any further purification. The samples of limestone were obtained from Nanjing Dou village. Mineralogical analysis and X-ray diffraction (XRD) showed that composition of the stone is composed of calcite (approximately 97\%), with trace amounts of quartz, alumina, and iron oxides. These stones were washed with water to remove the dust deposit, and stored in desiccators at $25^{\circ} \mathrm{C}$ and $50 \% \mathrm{RH}$ for $48 \mathrm{~h}$ prior to coating application. In addition, a part of limestone were crushed and screened to a No. 80 screen.

\subsection{Preparation of xerogel samples}

The hybrid sols were magnetically stirred for $10 \mathrm{~min}$ at room temperature by mixing TEOS with ethanol. Then, water was added under vigorous stirring, and 0\%, 3\%, 6\%, 9\%, 12\% and $17 \%$ (w/w) GPTMS was added drop by drop to the mixture under the same agitation. The magnetically stirring last to $2 \mathrm{~min}$. Subsequently, the mixture was under $10 \mathrm{~min}$ of exposure to ultrasonic agitation. Lastly, n-octylamine was added to each sol under vigorous stirring for $30 \mathrm{~min}$. The mole ratio of the TEOS/ $\mathrm{H}_{2} \mathrm{O} /$ ethanol/GPTMS/n-octylamine was 1:16:10:0.202-1.616:0.004. Hybrid monolithic xerogels were obtained as the following methods: ${ }^{12}$ sols of $10 \mathrm{ml}$ were cast in weighing bottle of $2.40 \mathrm{~cm}$ in diameter and 3.2 $\mathrm{cm}$ in length. Dried xerogels were obtained by simple exposure of the sols to laboratory conditions (relative humidity of $50 \%$ and temperature of $25^{\circ} \mathrm{C}$ ) until a constant weight was achieved. To simulate the solvent evaporation throughout the rock, it was limited by means of six diameter of 0.6 $\mathrm{mm}$ pinholes in the lid. Gel time was determined by a simple visualization of the gel transition inside the plastic centrifuge tube in the slow drying (tubes were sealed on top by lids that were perforated extensively with a needle) at $25^{\circ} \mathrm{C}$.

\subsection{Peeling performance and hardness tests}

Two milliliters sol was added to $5 \mathrm{~g}$ of limestone powder. The adherence of the coating to $5 \mathrm{~g}$ limestone powder samples surface was evaluated by performing a peeling test using 3 M Scotch magic Tape (translucent Scotch 810). The test was carried out according to previously reported methods. ${ }^{13}$ In total $3 \mathrm{M}$ transparent Scotch Tape was cut into pieces not less than $1 \mathrm{~cm}$ in length and with a contact surface area of about $1 \mathrm{~cm}^{2}$. The tapes were stuck to the surface and smoothed with gentle finger pressure, and were then removed by pulling at an angle of $90^{\circ}$, and weighed on a balance with sensitivity of $0.0001 \mathrm{~g}$. The peeled off material was determined as the difference between the weight of the tape after removal from the surface and the weight of the clean tape before application. The weight of the piece of Scotch Tape was calculated as an average of three pieces. The hardness of the hybrid sol to the $5 \mathrm{~g}$ limestone powders was measured using the LX-A hardness tester (Wuxi Xijing Instruments Ltd, China).

\subsection{Evaluation of protection against acid rain attack}

Three limestone samples were used to evaluate the protective ability of hybrid sol protective materials against acid rain attack. One sample was left untreated as blank. Another sample was treated on all faces with hybrid sol. For comparison, a further sample was treated with commercial fluoroorganosilane product (Mellerud) by brush. Samples were sprayed with $\mathrm{H}_{2} \mathrm{SO}_{4}$ solution circulated by a peristaltic pump as described previously. ${ }^{12}$ Each circulation lasted 8 h. $\mathrm{H}_{2} \mathrm{SO}_{4}$ solution used in the experiment was adjusted to $\mathrm{pH} 4.0$ to simulate acid rain. The total amount of solution volume was $1000 \mathrm{ml}$. In circulation, the volumetric flow rate of solution was arranged at $6 \mathrm{ml} \mathrm{min}-1$, and the rate was fixed for all samples. The flow rate of solution upon samples is very important parameter in this kind of experimental studies. The samples were placed at an angle of $45^{\circ}$ in a wide beaker and the solution was dropped on the samples continuously. The spray bottle was always placed at a controlled distance from the sample in order to cover the entire surface of the limestone sample. The $\mathrm{pH}$ of the run-off was controlled using a calibrated $\mathrm{pH}$ meter.

\subsection{Methods of characterization}

FTIR spectroscopy was recorded employing a Nicolet Nexus 870 FTIR spectrometer. A small amount of hybrid xerogels samples were mixed with $\mathrm{KBr}$ and pressed into pellets, then scanned from 4000 to $400 \mathrm{~cm}^{-1}$. The nitrogen adsorption-desorption isotherms were determined on an automatic adsorption apparatus (ASAP2010; micrometritics). The Brunauer-Emmett-Teller (BET) surface area was calculated from the linear part of the BET plot. Pore size distribution was calculated from desorption branch data according to the BJH method. Silica xerogels were also characterized in thermal behaviour using simultaneous differential thermal analysis (TA-SDT600), with a heating rate of $10^{\circ} \mathrm{C} \mathrm{min}^{-1}$ to $800{ }^{\circ} \mathrm{C}$ under flowing nitrogen $(100$ $\left.\mathrm{ml} \min ^{-1}\right)$. Clean mica were dipped completely in the sols under study and dried under laboratory conditions. The surface roughness of the coatings was investigated using atomic force microscopy (AFM, Agilent Technology 5500) operated in tapping mode. Roughness measurements were performed on $5 \times 5 \mu^{2}$ scans. The contact angles were measured with a $5 \mu \mathrm{l}$ water droplet at ambient temperature with an optical contact angle meter (DSA100 of KRUSS Instruments Ltd, Germany). The reported values of contact angles were averages of five measurements made on different points of the sample surface. Calcium ions in circulated acid rain solution were determined by atomic absorption spectrophotometer (Varian AA240). The viscosity of sol was determined by NDJ-5S viscometer (Shanghai Pingxian Instruments Ltd, China). 


\section{Results and discussion}

\subsection{Characterization of gels obtained in vitro}

As an important parameter closely related with the consolidant penetration into the stone, viscosity of each sol prepared is determined at $25^{\circ} \mathrm{C}$. To compare the viscosity of various sols, the viscosity is immediately measured after GPTMS addition. The effect of the addition of GPTMS to the sols on the sol viscosity is shown in figure 1 . A relatively small increase in viscosity depending on the amount of GPTMS was observed. It can be seen that sol viscosity gradually increases from $1.75 \mathrm{mPa} \mathrm{s}^{-1}$ to $2.41 \mathrm{mPa} \mathrm{s}^{-1}$ with the addition of GPTMS since viscosity of GPTMS is $3.4 \mathrm{mPa} \mathrm{s}^{-1}$. Thus, the ability to penetrate into stone substrates would remain practically unaffected since the viscosity of the commercial consolidant is commonly $2-3.3 \mathrm{mPa} \mathrm{s}^{-1} .{ }^{14}$ Figure 2 shows gel time change with GPTMS concentration. The gel

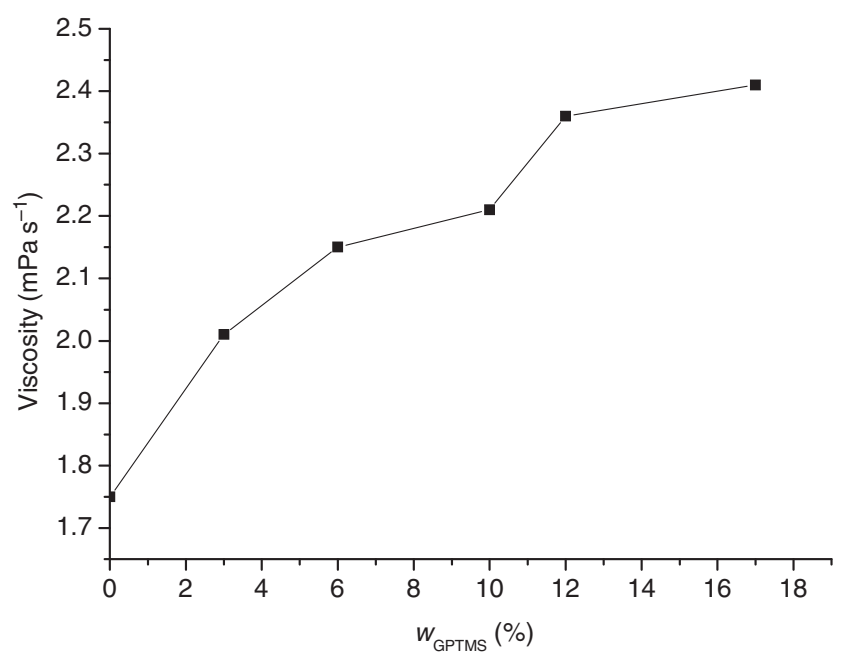

Figure 1. Effect of GPTMS content on viscosity $\left(25^{\circ} \mathrm{C}\right)$.

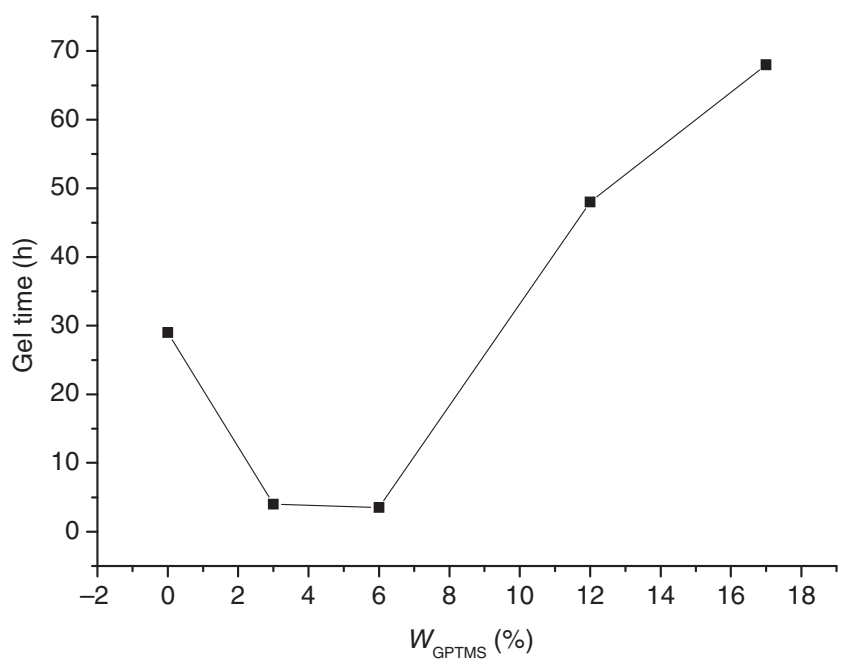

Figure 2. Effect of GPTMS content on gel time $\left(25^{\circ} \mathrm{C}\right)$. time decreases first and then increases with the increase in the concentrations of GPTMS. This behavior can be explained because GPTMS chains in the starting sol contain the most silanol terminals which must be hydrolyzed as a prior stage to the gel transition, therefore, the gel time decreases when GPTMS was added. The gel time increases when GPTMS is further added because the epoxide ring of GPTMS can react with $n$-octylamine which is one of the factors affecting the gel time. When GPTMS is mixed with TEOS, three different condensation processes may occur: (1) co-condensation between two components, (2) self-condensation of GPTMS or (3) hydrolysis of TEOS and the subsequent condensation of silanols. Thus, it is clear that the amount of GPTMS will determine the structure of the final product. Therefore, the final properties of the composites depend on the morphology of the two component phases in the hybrid material and on their mutual interaction. After drying under laboratory conditions, the xerogels synthesized in the presence of surfactant gave homogeneous monolithic xerogels. Colour of hybrid xerogels became translucent or opaque (figure 3) with the addition of GPTMS.

FTIR transmittance spectra of the xerogels under study is shown in figure 4. For all TEOS/GPTMS hybrid gel sample studied here, there is a very pronounced band appearing at $460,798,1078 \mathrm{~cm}^{-1}$ which corresponded to the absorption of $\mathrm{Si}-\mathrm{O}-\mathrm{Si}$ groups, indicating that hybrid gel samples are mainly composed of a silica network. ${ }^{10}$ The broad band centred at $3427 \mathrm{~cm}^{-1}$ and the sharp band at $1631 \mathrm{~cm}^{-1}$ are, respectively, assigned to the $\mathrm{O}-\mathrm{H}$ stretching and bending absorptions. ${ }^{15}$ The weak bands at 563, 763, $912 \mathrm{~cm}^{-1}$ attributed to antisymmetric epoxide ring bands. ${ }^{7}$ Two bands at 2937 and $2869 \mathrm{~cm}^{-1}$ are assigned to stretching and bending of the $\mathrm{C}-\mathrm{H}$ bond, respectively. The peak at $1251 \mathrm{~cm}^{-1}$ is the band of the epoxy ring breathing can be clearly seen growing with increasing GPTMS content in the spectra, since the amount of $n$-octylamine is smaller compared with that of GPTMS, although the epoxide ring is readily opened by amines. $^{16-18}$

Textural properties of the xerogels under study are determined from the nitrogen adsorption-desorption isotherms (figure 5) and are given in table 1 . The three xerogels $(0 \%$, $3 \%, 6 \%$ ) synthesized in the presence of the surfactant show type IV isotherms, which are typical of mesoporous materials, according to the IUPAC classification while the xerogel synthesized (17\%) indicates adsorption in micropores. Concerning the effects on textural properties for three xerogels, pore size decreases with the addition of GPTMS. Furthermore, change of pore volume is also similar. The BJH pore size distribution for the materials under study is shown in figure 6 . The silica exhibited a narrow pore size distribution $5-10 \mathrm{~nm}$ without the addition of GPTMS, which is a clear indication of the role played by $n$-octylamine in directing the formation of the pore structure. ${ }^{8}$ The most adsorption of xerogel $(17 \%)$ takes place at low relative pressure values, which indicates that the pore size in the network is small. As the capillary pressure supported by the gel network during drying is inversely proportional to the gel pore size, the 

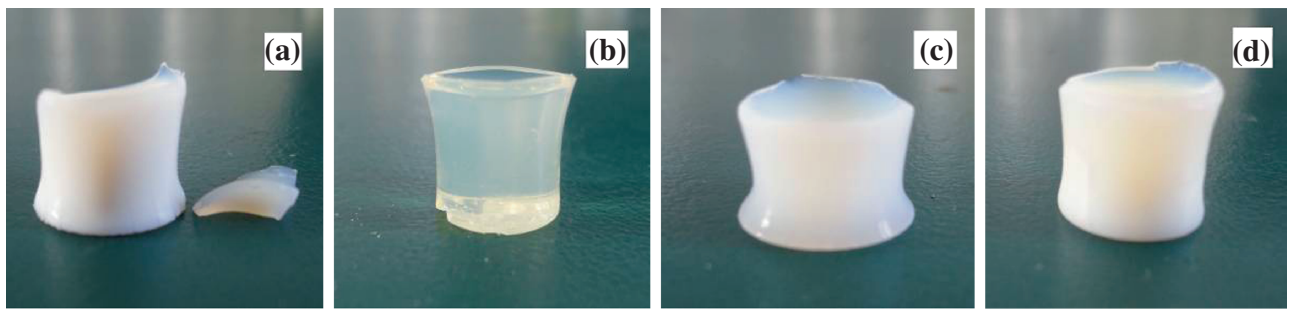

Figure 3. Hybrid xerogels derived from TEOS-GPTMS after drying under laboratory conditions ((a) $0 \%,(\mathbf{b}) 3 \%,(\mathbf{c}) 6 \%$ and (d) $9 \%$ ).

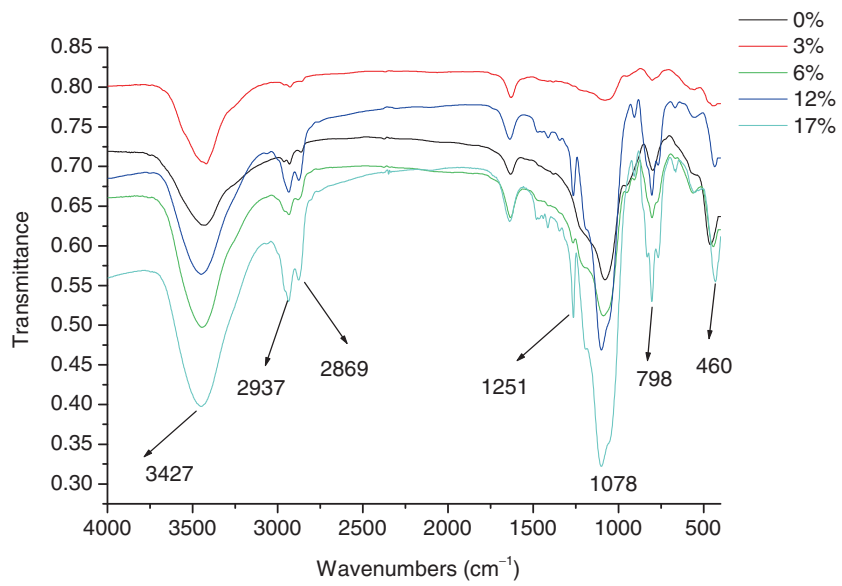

Figure 4. FTIR spectra of the TEOS/GPTMS xerogels under study.

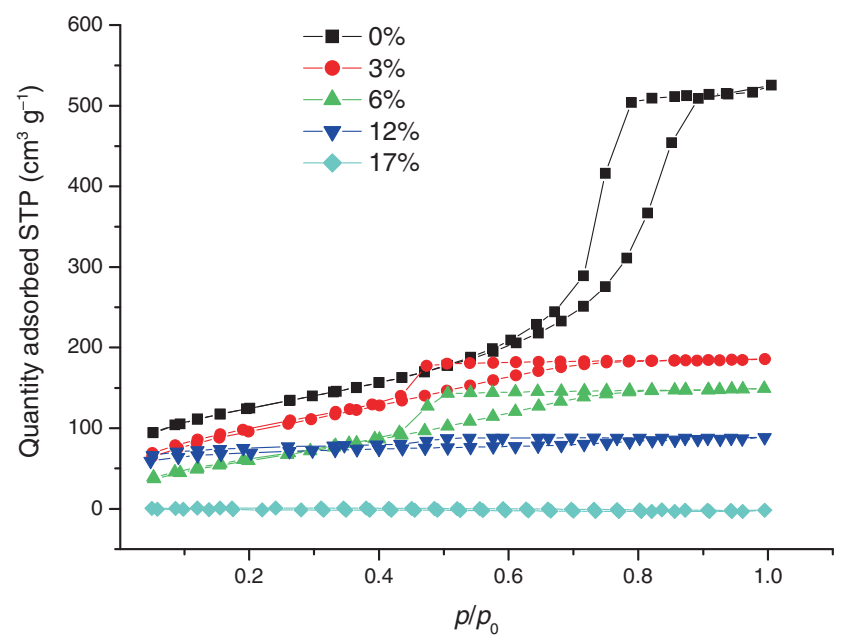

Figure 5. Nitrogen adsorption isotherms for the xerogels under study.

decreasing of the gel pores size clearly promotes a higher capillary pressure during drying. Therefore, the flexible segment of silica xerogels containing GPTMS, responsible for their crack-free, was explained by Kim et al. ${ }^{7}$

Figure 7 shows the 3D images of the surfaces. Surfaces of the coatings without GPTMS presented a slight roughness. Surfaces of the silica nanomaterials without PDMS presented
Table 1. Gel properties of TEOS-GPTMS hybrid.

\begin{tabular}{llcc}
\hline & \multicolumn{3}{c}{ Gel properties } \\
\cline { 2 - 4 } Sample & $\begin{array}{c}\text { Pore volume } \\
\left(\mathrm{cm}^{3} \mathrm{~g}^{-1}\right)\end{array}$ & $\begin{array}{c}\text { ABET surface } \\
\left(\mathrm{m}^{2} \mathrm{~g}^{-1}\right)\end{array}$ & $\begin{array}{c}\text { Average pore } \\
\text { diameter }(\mathrm{nm})\end{array}$ \\
\hline TEOS /0\% GPTMS & 0.81 & 449.7 & 7.2 \\
TEOS/3\% GPTMS & 0.29 & 273.6 & 4.2 \\
TEOS/6\% GPTMS & 0.23 & 233.2 & 4.0 \\
TEOS/12\% GPTMS & 0.061 & 241.9 & 2.2 \\
TEOS/17\% GPTMS & 0.0014 & 2.923 & 1.8 \\
\hline
\end{tabular}

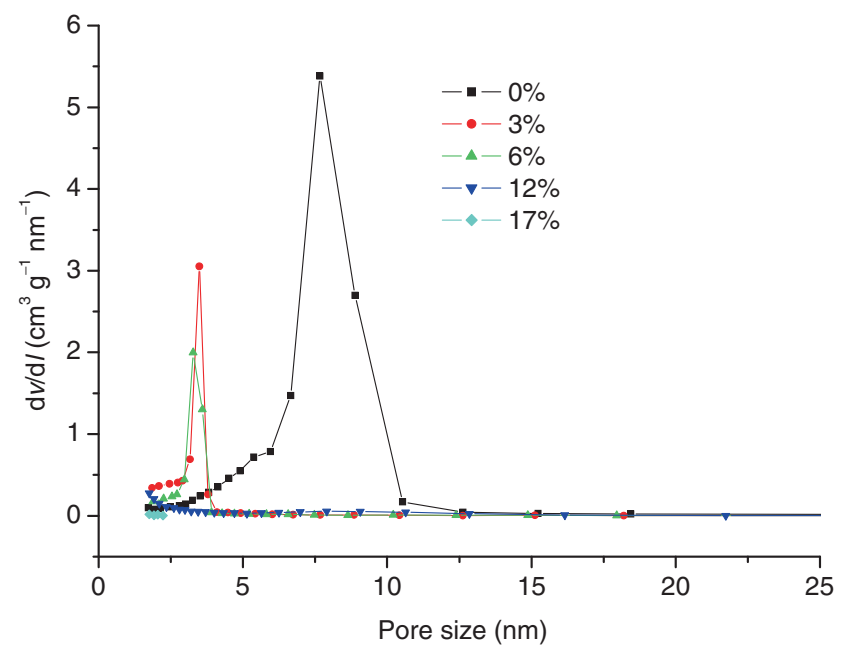

Figure 6. Pore size distribution for the TEOS/GPTMS hybrid xerogels.

a slight roughness, which was associated with the aggregation of the silica particles. Addition of GPTMS substantially modified the surface morphology of the coatings: peaks appear that greatly increase their surface roughness. These peaks may be associated with the GPTMS chains. Surface roughness parameters were calculated for the overall AFM image. ${ }^{19}$ The coatings roughness is $0.16 \mathrm{~nm}$ without addition of GPTMS, while the roughness is $1.29 \mathrm{~nm}$ when concentration of GPTMS is $6 \%$. Roughness of the coating increases as GPTMS content is higher. The coatings roughness is 14.9 $\mathrm{nm}$ when concentration of GPTMS is $12 \%$.

TGA analysis (figure $8 \mathrm{a}$ ) shows that, at $800^{\circ} \mathrm{C}$, all samples have completely lost both the water and the solvent adsorbed 

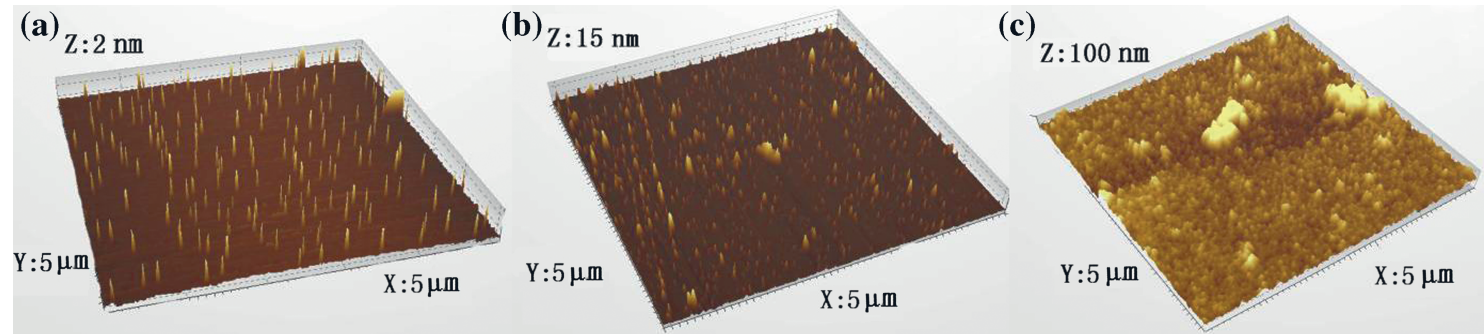

Figure 7. AFM 3D topography images of the coatings deposited on mica ((a) $0 \%,(\mathbf{b}) 6 \%$ and (c) $12 \%)$.

(a)

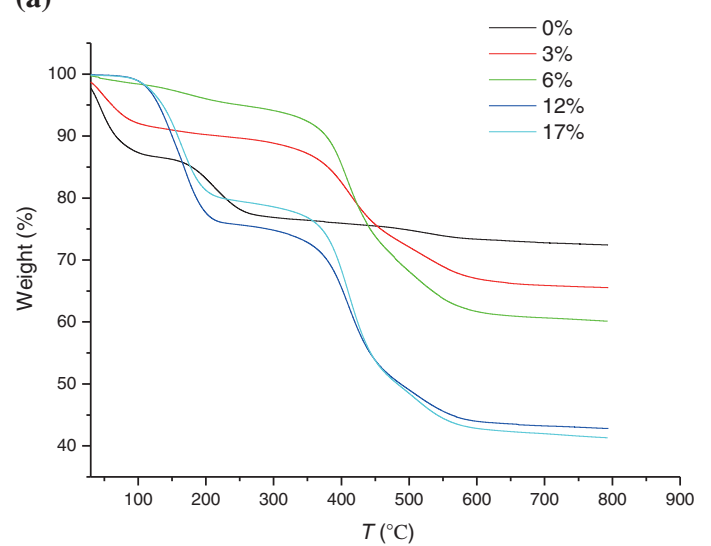

(b)

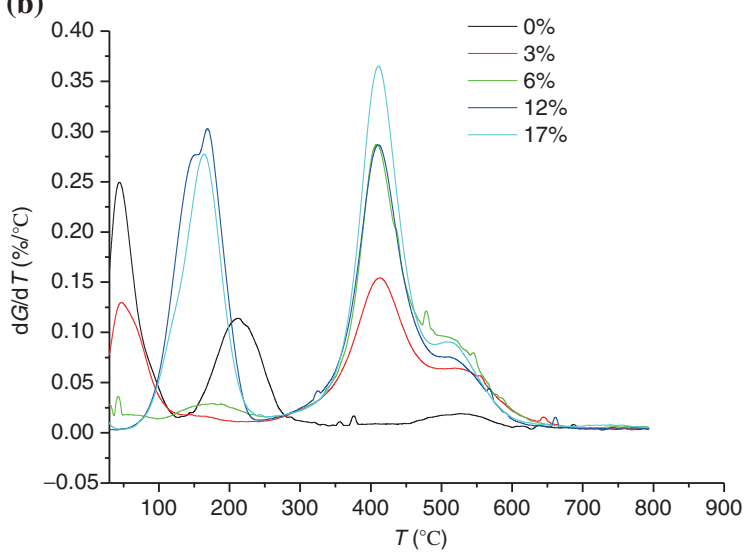

Figure 8. TGA analysis for hybrid xerogels ((a) TGA curve and (b) derivative of TGA curve).

in the structure, as well as the unreacted alkoxy groups, leaving behind only the total amount of $\mathrm{SiO}_{2}$ formed. This concept is known as ceramic yield. ${ }^{20}$ The xerogel synthesized without GPTMS presented the highest percentage of silica formed in the structure ( $72.4 \%$ of ceramic yield), whereas only $41.3 \%$ is obtained when concentration of GPTMS is $17 \%$. This is due to the increasing of propyl groups of the GPTMS which are eliminated with the addition of GPTMS. The DSC-TGA study for $12 \%$ GPTMS is shown in figure $8 \mathrm{~b}$ as an example of the thermal behaviour of these types of materials. TGA derivative analysis for hybrid xerogels shows one endothermic peak (around $168^{\circ} \mathrm{C}$ ), which is assigned to unreacted alkoxy groups. A second, exothermic peak $\left(410^{\circ} \mathrm{C}\right)$ is assigned to the gradual disappearance of the propyl groups. The hybrid xerogels in the absence of GPTMS have a much higher thermal stability as evidenced by a higher decomposing temperature at $209^{\circ} \mathrm{C}$. It is clear that the presence of the GPTMS in the hybrid xerogels results in a lower thermal stability.

The treated limestone powder shows homogeneous columniform solid. The hardness value of the limestone powders before treatment is 0 HA. After treatment with hybrid sol, hardness is enhanced (see table 2). A greater increase in hardness is observed when hybrid sol $(6 \%)$ is applied, the hardness values are $30 \mathrm{HA}$. The hardness values increases with the concentration of GPTMS. The maximum hardness values are $65 \mathrm{HA}$ when concentration of GPTMS is $17 \%$. Typical peeling test results for the individual limestone
Table 2. Properties of calcite power with hybrid sol.

\begin{tabular}{lcc}
\hline & Hardness & $\begin{array}{l}\text { Loss of material by } \\
\text { peeling }\left(\mathrm{mg} \mathrm{cm}^{-2}\right)\end{array}$ \\
\hline Untreated & 0 & 7.8 \\
TEOS /0\% GPTMS & 26 & 5.4 \\
TEOS/6\% GPTMS & 30 & 1.9 \\
TEOS/12\% GPTMS & 40 & 1.7 \\
TEOS/17\% GPTMS & 65 & 1.5 \\
\hline
\end{tabular}

powders are shown in table 2. Results of the peeling performance tests results indicate a consistent increase in cohesion after the application of hybrid sol to those powder samples that previously exhibited poor cohesion.

\subsection{Characterization of gels obtained in situ}

The contact-angle measurement was used to evaluate the overall effectiveness of hybrid materials by considering the hydrophobicity of surfaces which is then attributed to the values of contact-angle equal to or higher than $90^{\circ}$. Figure 9 shows the contact angle $(\theta)$ values for various concentrations of GPTMS measured using the contact angle meter. The mean $\theta$ values were $73^{\circ}$ for untreated limestone while $\theta$ values of limestone treated with hybrid sol increased first, reached the maximum $\left(123^{\circ}\right)$, and then decreased. The 


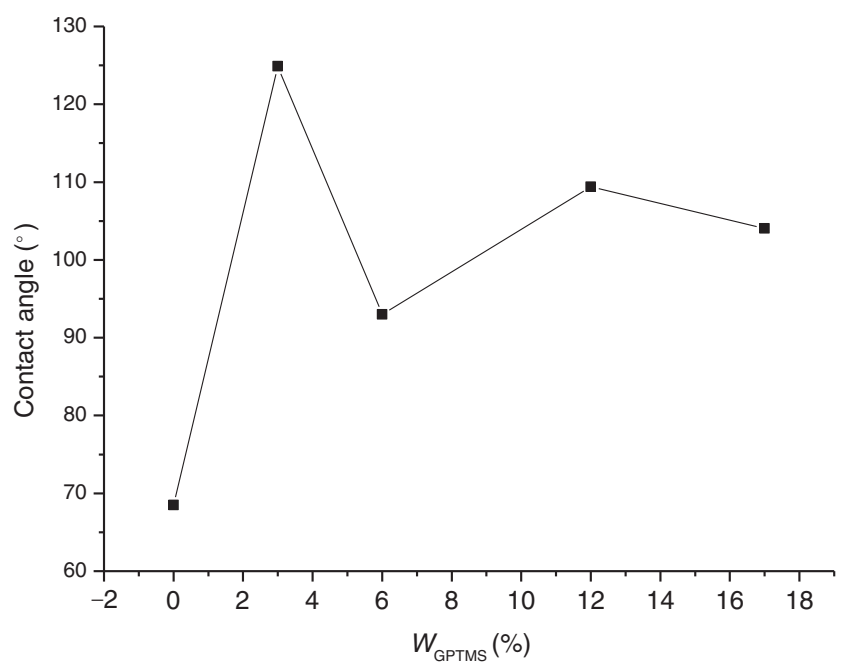

Figure 9. Values of contact angle for various concentrations of GPTMS.

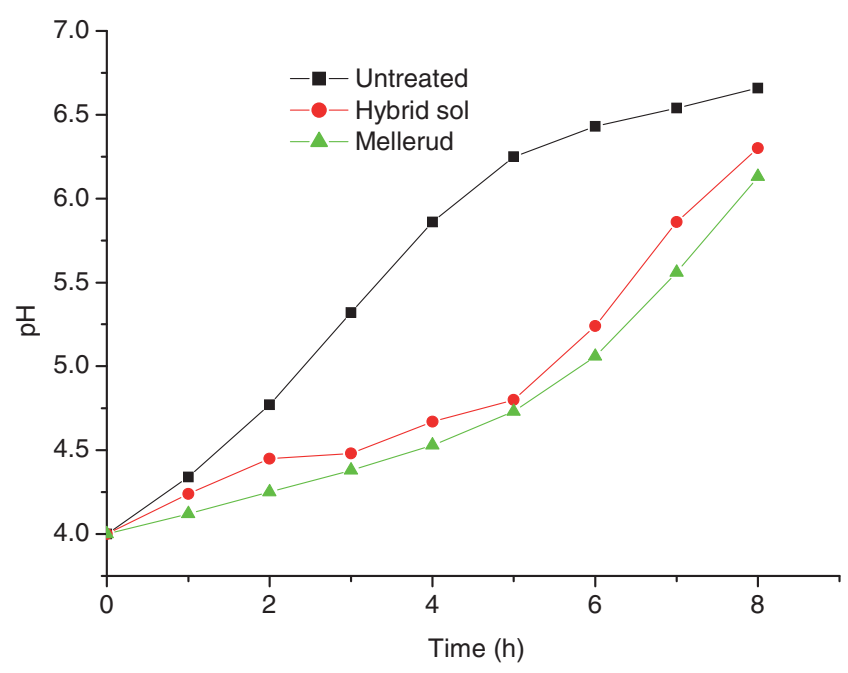

Figure 10. $\mathrm{pH}$ change of the solution with washing time.

hybrid sol treatment induced a considerable increase of contact angle on the carbonate stone, which was attributed to the increased organic content of the hybrid gels. The change of contact angle with the addition of GPTMS was probably caused by the surface morphology alteration, ${ }^{21-23}$ since hybrid sol can change the surface morphology of stone.

Integrated hydrophobicity, hardness and peeling performance, the limestone treated with hybrid sol $\left(\mathrm{w}_{\text {GPTMS }} \%\right.$ $=12 \%$ ) were used to evaluate the protective ability against acid rain attack. The $\mathrm{pH}$ variation of the run-off from the carbonate stone with time during exposure to acid rain solution of initial $\mathrm{pH} 4$ is presented in figure 10. For untreated sample, the run-off $\mathrm{pH}$ is close to 7 after exposure for $8 \mathrm{~h}$ and remains approximately constant. The trend of concentration of calcium ions in solution is accord with that of $\mathrm{pH}$, which is shown in figures 10 and 11. It is noticeable in the graph (figures 10 and 11) that the $\mathrm{pH}$ value and calcium ions

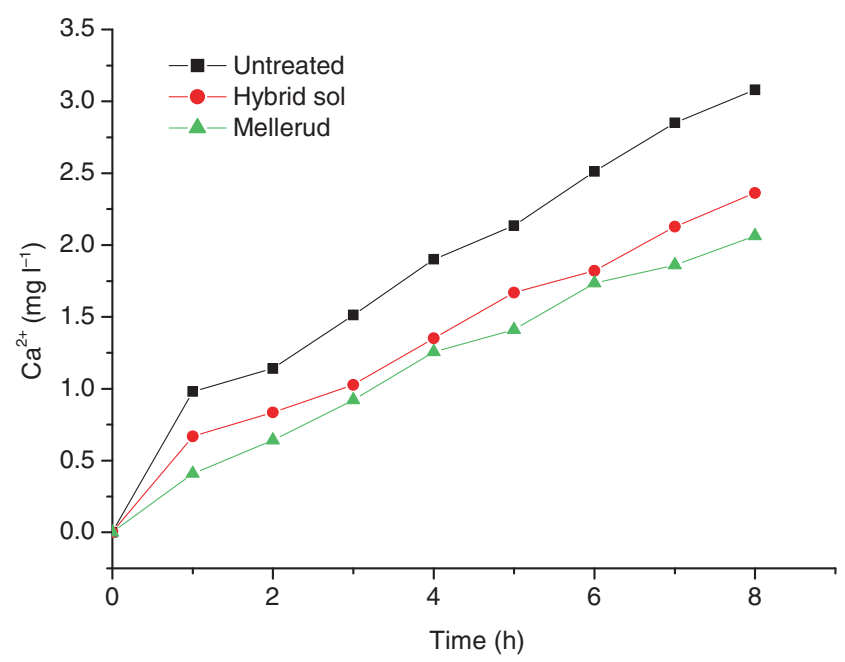

Figure 11. $\mathrm{Ca}^{2+}$ change of the solution with washing time.

concentration is higher for untreated sample than the treated samples at the same time, and the protective efficiency of the hybrid sol is similar to the commercial product. It is a fact that $\mathrm{Ca}^{2+}$ could be measured possibly due to the incomplete blocking of hybrid sol on stone holes which allows the acid rain leading a corrosion through entering the stone holes.

\section{Conclusions}

Monolithic xerogels were obtained by using a surfactant $n$-octylamine as a catalyst containing TEOS and GPTMS as an additive. TGA analysis shows ceramic yield decreases with the addition of GPTMS and the presence of the GPTMS in the hybrid xerogels results in a lower thermal stability. With the addition of the GPTMS, the pore size of silica becomes small. GPTMS as flexible segment contribute to gel crack-free. AFM images showed clearly that the GPTMS content modifies the surface morphology of the coating, roughness being increased as GPTMS content is higher. The Scotch Tape test and the hardness values show improvement of cohesion and consolidation ability of hybrid sol. The protective performance evaluation of the treated stones with hybrid sol indicated its acid rain resistance.

\section{Acknowledgements}

This work was supported by the Foundation of the Cultural Heritage Conservation Science and Technology of National Heritage Board (20110131), and School Foundation of Nanchang University (Z04860).

\section{References}

1. Xu F, Li D, Chen W and Gao S 2010a Chin. J. Chem. 281487

2. Xu F, Tang J and Gao S 2010b J. Cult. Heri. 11232 
3. Sacchi B, Giannini L, Frediani M, Rosi L and Frediani P 2013 J. Coat. Technol. Res. 10649

4. Mosquera M J, Pozo J and Esquivias L 2003 J. Sol-Gel Sci. Technol. 261227

5. Scherer G W 1990 J. Am. Ceram. Soc. 733

6. Son S, Won J, Kim J J, Jang Y D, Kang Y S and Kim S D 2009 ACS Appl. Mater. Interfaces 1393

7. Kim E K, Won J and Do J Y 2009 J. Cult. Heri. 10214

8. Mosquera M J, De los Santos D M, Valdez-Castro L and Esquivias L 2008 J. Non-Cryst. Solids 354645

9. Mosquera M J, De los Santos D M and Rivas T 2010 Langmuir 266737

10. Pinho L and Mosquera M J 2011 J. Phys. Chem. C 11522851

11. Illescas J F and Mosquera M J 2012 ACS Appl. Mater. Interfaces 44259

12. Xu F, Li D, Zhang H and Peng W 2012 J. Sol-Gel Sci. Technol. 61429

13. Drdácký M, Lesák J, Rescic S, Slî’ źková Z, Tiano P and Valach J 2012 Mater. Struc. 45505
14. Salazar-Hern'andeza C, Puy Alquizab M J, Salgadoa P and Cervantesa J 2010 Appl. Organometal. Chem. 24481

15. Fidalgo A, Ciriminna R, Iharco L M and Pagliaro M 2005 Chem. Mater. 176686

16. Khramov A N, Balbyshev V N, Voevodin N N and Donley M S 2003 Prog. Org. Coat. 47207

17. Vreugdenhil A J, Gelling V J, Woods M E, Schmelz J R and Enderson B P 2008 Thin Solid Films 517538

18. Cardiano P, Sergi S, Lazzari M and Piraino P 2002 Polymer 436635

19. Kiely J D and Bonnell D A 1997 J. Vac. Sci. Technol. B 15 1483

20. Salazar-Hernańdez C, Zárraga R, Alonso S, Sugita S, Calixto S and Cervantesa J 2009 J. Sol-Gel Sci. Technol. 49301

21. Toniolo L, Poli T, Castelvetro V, Manariti A, Chiantore O and Lazzari M 2002 J. Cult. Heri. 3309

22. Coulson S R, Woodward I, Badyal J P S, Brewer S A and Willis C 2000 J. Phys. Chem. B 1048836

23. Zielecka M and Bujnowska E 2005 Prog. Org. Coat. 55160 